Original Article

\title{
Effectiveness of physical activity as primary preventive care for lower urinary tract symptoms in elderly people through the "Muscle Enhancing Club"
}

\author{
Seiji Matsumoto, MD, $\mathrm{PhD}^{1)}$ \\ 1) Clinical Research Support Center, Asahikawa Medical University Hospital: \\ 2-1-1-1 Midorigaoka-Higashi, Asahikawa, Hokkaido 078-8510, Japan
}

\begin{abstract}
Purpose] The aim of this study was to examine the relationship between physical activity (PA) in elderly people as preventive exercise for lower urinary tract symptoms (LUTS) or overactive bladder (OAB), and how PA in primary preventive care can impact change upon LUTS. [Subjects and Methods] An interview sheet featuring LUTS domains (IPSS/QOL and OABSS questionnaire) was distributed to all participants (104 males and 494 females) who attended the public elderly people's physical exercise class "Muscle Enhancing Club 2010" in Asahikawa city. [Results] The interview sheet was collected from 65 males ( $75.4 \pm 5.79$ years of age) and 304 females (72.7 \pm 5.23 years of age). In all cases, there was a statistically significant difference of QOL. In 77 LUTS cases, there was a statistically significant difference in the change of the following LUTS domains: incomplete bladder emptying, frequency, nocturia, QOL, urgency, and OABSS total score. In 61 OAB cases, there was a statistically significant difference in the change of the following LUTS domains: QOL, urgency, and OABSS total score. [Conclusion] PA, as a preventative exercise for elderly people, led to improvements in LUTS, especially storage symptoms and QOL. Our data show that gross movement and education was beneficial. From the point of preventive medicine, various exercise classes, organized by the local government and others, also indicated that these classes increase the possibility of improvement to LUTS.

Key words: Lower urinary tract symptoms, Physical activity, Primary preventive care
\end{abstract}

(This article was submitted Feb. 20, 2017, and was accepted Apr. 17, 2017)

\section{INTRODUCTION}

Several reports have shown that bladder training (BT), pelvic floor muscle training (PFMT), and other types of training are well known as exercise therapy. In particular, physiotherapy (physical therapy) involving urination leads to improvements in lower urinary tract symptoms (LUTS), including stress urinary incontinence (SUI) and overactive bladder (OAB) $)^{1-3)}$. However, these physical activities (PA) represent local motion, not gross movement. Physiotherapy in elderly people has attracted attention by the booming healthy life style and the development of preventive medicine ${ }^{4,5)}$; however, there has been little attention paid to the effect of this practice on LUTS. If we can prove that PA in elderly people, as part of primary preventive care program, can improve LUTS, it would bring significant meaning to the viewpoint of preventive medicine and also various public preventive exercise classes. To our knowledge, the relationship between PA in elderly people as a preventive exercise for LUTS has never been studied. Therefore, the aim of this study was to examine the relationship between PA in elderly people, as part of a preventive exercise, and LUTS or OAB, and how this practice can influence changes in LUTS. To do this, we focused our attention upon the "Muscle Enhancing Club 2010" in Asahikawa city. 


\section{SUBJECTS AND METHODS}

The interview sheet of LUTS domains (International Prostate Symptom Score (IPSS)/QOL and OAB Symptom Score (OABSS) questionnaire) was distributed to all participants (104 males; $75.8 \pm 5.63$ years of age; and 494 females; $73.0 \pm$ 5.24 years of age) who attended the public elderly physical exercise class "Muscle Enhancing Club 2010" in Asahikawa city, Hokkaido, Japan. The "Muscle Enhancing Club" is an exercise class, which forms part of a primary preventative care program in Asahikawa city, and provides a PA class for senior people (over 65 years of age with no certification of long-term care need). The "Muscle Enhancing Club" includes the following movement upgrade programs: aerobic exercise, stretch, balance movement, muscle strength exercise, talking about health, and physical fitness tests. These are all provided within one course (a 1.5-hour class, once a week for 12 weeks).

This study was approved by Asahikawa City and Asahikawa Medical University Ethical Committee (No. 820). The purpose of this study and the procedures involved were explained to all subjects, who provided written informed consent prior to participation. The interview sheets were collected before and after patients attended the 'Muscle Enhancing Club 2010'. We included participants who completed the interview questionnaire. We excluded men who did not fill the interview questionnaire. We examined the relationship between PA in elderly people, as a preventive exercise for LUTS or OAB as follow: [1] The change of LUTS domains in all participants, [2] The change of domains in participants with LUTS (+), [3] The change in domains of the participants with $\mathrm{OAB}(+)$, and [4] The change in domains in the participants who were LUTS $(-)$ and $\mathrm{OAB}(-)$.

The IPSS questionnaire ${ }^{6}$ ) was used to detect LUTS and assess its severity. This questionnaire features the following seven questions (Q) pertaining to specific symptoms: feeling of incomplete bladder emptying (Q1), frequency (Q2), intermittency (Q3), urgency (Q4), weak stream (Q5), straining (Q6) and nocturia (Q7), each referring to the previous month, and each involving the assignment of a score from 1 to 5 , for a total of 35 points maximum. The QOL was assigned a score of 1 to 6 (Total score: 0-7 mild; 8-19 moderate; and 20-35 severe symptoms). LUTS (+) was defined as individuals having a total score of IPSS $\geq 8$ and a QOL score $\geq 2$.

The OABSS questionnaire ${ }^{7)}$ was used to detect OAB and assess its severity. The OABSS is a symptom assessment tool designed to combine $\mathrm{OAB}$ symptoms into a single score. It consists of the following four questions (Q) regarding specific symptoms: daytime frequency $(\mathrm{Q} 1)$, night-time frequency $(\mathrm{Q} 2)$, urgency $(\mathrm{Q} 3)$, and urgency incontinence (Q4). Patients are asked to rate their symptom severity on a scale with a maximum (worst) score of 2, 3, 5 and 5 , respectively. According to the clinical guidelines for $\left.\mathrm{OAB}^{8}\right), \mathrm{OAB}(+)$ was defined as urinary urgency once a week or more $(\mathrm{Q} 2 \geq 2)$ and total score of $\mathrm{OABSS} \geq 3$. The total score for the OABSS ranges from 0 to 15 , with higher scores indicating increasing symptom severity ( 5 or less, mild; 6-11, moderate; 12 or more, severe). Statistical analyses were performed using the paired t-test except for missing data. Statistical analyses were performed using SPSS version 15.0, and statistical significance was set at $\mathrm{p}<0.05$.

\section{RESULTS}

Interview sheets were collected from 65 males (65-89; median 75, 75.4 \pm 5.79 years of age) and 304 females (64-91; median $72,72.7 \pm 5.23$ years of age $)$. The response rate was $61.7 \%(\mathrm{M} / \mathrm{F}=62.5 / 61.5 \%)$.

When investigating the changes in LUTS domains (IPSS/QOL and OABSS) amongst all participants (Table 1), we found that the only significant QOL difference was based on gender differences. Only female participants showed any significant difference in terms of changes in the various LUTS domains. However, when we investigated the changes in domains only in the participants with LUTS $(+)$ (Table 2), we found that LUTS $(+)$ cases involved 77 people $(20.9 \%)$ with a mean age of 73.9 years. Of these, 19 were male $(29.2 \%$ with a mean age of 75.0 years), and 58 were female $(11.9 \%$ with a mean age of 73.5 years). There were statistically significant differences in the change of LUTS domains for incomplete bladder emptying (Q1 of IPSS), frequency (Q2 of IPSS), nocturia (Q7 of IPSS), QOL, urgency (Q3 of OABSS) and OABSS total.

When we investigated LUTS domains in participants with OAB $(+)$ (Table 2), we identified 61 OAB $(+)$ cases $(16.6 \%)$ with a mean age of 75.2 years. Seven of these were male with a mean age of 74.7 years, and 54 were female (11.1\%) with a mean age of 75.2 years. There was a statistically significant difference in the change of LUTS domains for QOL in terms of urgency (Q3 of OABSS) and OABSS total.

Finally, we investigated the change in LUTS domains (IPSS/QOL and OABSS) when considering participants who were LUTS (-) and OAB (-) (Data not shown). There was no statistically significant difference in the change of LUTS domains for this group of participants. Because of PA, via a preventative exercise class, the participants who were LUTS $(+)$ and OAB $(+)$ showed improvement in LUTS, particularly in terms of storage symptoms, but also gross movement.

\section{DISCUSSION}

Bladder training (BT), pelvic floor muscle training (PFMT), and other types of physiotherapy are known to improve lower urinary tract symptoms (LUTS), including stress urinary incontinence (SUI) and overactive bladder (OAB) ${ }^{1-3)}$. As a result, BT and PFMT should be considered as the first-line choice for the initial treatment of OAB. The primary mechanism underly- 
Table 1. The change of LUTS domains when analyzed across all participants

\begin{tabular}{|c|c|c|c|c|c|c|c|}
\hline & & \multicolumn{2}{|r|}{ Total } & \multicolumn{2}{|r|}{ Male } & \multicolumn{2}{|c|}{ Female } \\
\hline & & $\mathrm{N}$ & Mean \pm SD & $\mathrm{N}$ & Mean \pm SD & $\mathrm{N}$ & Mean \pm SD \\
\hline \multirow[t]{8}{*}{ IPSS } & 1. Incomplete emptying & 364 & $-0.06 \pm 0.98$ & 65 & $-0.20 \pm 0.97$ & 299 & $-0.03 \pm 0.98$ \\
\hline & 2. Frequency & 353 & $-0.06 \pm 1.20$ & 63 & $-0.03 \pm 1.06$ & 290 & $-0.07 \pm 1.23$ \\
\hline & 3. Intermittency & 361 & $0.06 \pm 1.05$ & 65 & $0.28 \pm 1.14$ & 296 & $0.01 \pm 1.03$ \\
\hline & 4. Urgency & 359 & $0.05 \pm 1.16$ & 65 & $-0.06 \pm 1.26$ & 294 & $0.07 \pm 1.14$ \\
\hline & 5. Weak stream & 359 & $0.06 \pm 1.27$ & 64 & $-0.03 \pm 1.26$ & 295 & $0.08 \pm 1.28$ \\
\hline & 6. Straining & 361 & $0.04 \pm 1.16$ & 64 & $0.00 \pm 1.91$ & 297 & $0.05 \pm 0.92$ \\
\hline & 7. Nocturia & 352 & $-0.03 \pm 0.65$ & 64 & $-0.02 \pm 0.70$ & 288 & $-0.04 \pm 0.64$ \\
\hline & Total score & 367 & $0.04 \pm 4.25$ & 65 & $-0.15 \pm 4.50$ & 302 & $0.08 \pm 4.21$ \\
\hline \multicolumn{2}{|c|}{ IPSS/QOL } & 355 & $-0.27 \pm 1.36^{*}$ & 64 & $-0.22 \pm 1.11$ & 291 & $-0.28 \pm 1.41^{*}$ \\
\hline \multirow[t]{5}{*}{ OABSS } & 1. Frequency & 360 & $-0.00 \pm 0.56$ & 62 & $0.11 \pm 0.52$ & 298 & $-0.03 \pm 0.56$ \\
\hline & 2. Nocturia & 360 & $-0.01 \pm 0.53$ & 61 & $0.03 \pm 0.55$ & 299 & $-0.02 \pm 0.53$ \\
\hline & 3. Urgency & 362 & $-0.05 \pm 0.97$ & 62 & $-0.08 \pm 1.04$ & 300 & $-0.04 \pm 0.98$ \\
\hline & 4. Urge incontinence & 361 & $-0.04 \pm 0.77$ & 62 & $-0.06 \pm 0.62$ & 299 & $-0.04 \pm 0.79$ \\
\hline & Total score & 363 & $-0.10 \pm 1.69$ & 62 & $0.05 \pm 1.80$ & 301 & $-0.14 \pm 1.67$ \\
\hline
\end{tabular}

LUTS domains questionnaire with IPSS/QOL and OABSS

$*_{\mathrm{p}}<0.05$ analyzed by paired-t test

Table 2. The change of LUTS domains in participants who were classified as LUTS $(+)$ and $\mathrm{OAB}(+)$

\begin{tabular}{llccc}
\hline & \multicolumn{2}{c}{ LUTS $(+)(\mathrm{N}=77)$} & & OAB $(+)(\mathrm{N}=61)$ \\
\cline { 3 - 3 } IPSS & Mean \pm SD & & Mean \pm SD \\
\cline { 5 - 5 } & 1. Incomplete emptying & $-0.67 \pm 1.41^{*}$ & & $-0.21 \pm 1.02$ \\
& 2. Frequency & $-0.64 \pm 1.66^{*}$ & & $-0.26 \pm 1.58$ \\
& 3. Intermittency & $-0.11 \pm 1.79$ & & $0.24 \pm 1.64$ \\
& 4. Urgency & $-0.22 \pm 1.93$ & & $0.09 \pm 1.82$ \\
& 5. Weak stream & $-0.37 \pm 1.68$ & & $-0.18 \pm 1.56$ \\
& 6. Straining & $-0.03 \pm 1.92$ & & $0.12 \pm 1.55$ \\
& 7. Nocturia & $-0.15 \pm 0.61^{*}$ & & $0.00 \pm 0.64$ \\
& Total score & $-2.20 \pm 5.85^{*}$ & & $-0.28 \pm 5.01$ \\
\hline IPSS/QOL & & $-0.47 \pm 1.27^{*}$ & & $-0.59 \pm 1.51^{*}$ \\
\hline OABSS & 1. Frequency & $-0.01 \pm 0.48$ & & $-0.04 \pm 0.63$ \\
& 2. Nocturia & $-0.03 \pm 0.43$ & & $0.03 \pm 0.49$ \\
& 3. Urgency & $-0.41 \pm 1.36^{*}$ & & $-0.97 \pm 1.37^{*}$ \\
& 4. Urge incontinence & $-0.19 \pm 0.87$ & & $-0.25 \pm 1.08$ \\
& Total score & $-0.63 \pm 2.03^{*}$ & $-1.23 \pm 2.23^{*}$ \\
\hline
\end{tabular}

LUTS $(+)$ was defined as when total IPSS 8 and when QOL 2. OAB (+) was defined as when OABSS Q3 2 and when OABSS total 3. ${ }^{*} \mathrm{p}<0.05$ analyzed by paired-t test

ing the efficacy of these treatments is improvement in the inhibitory function of bladder contraction in line with the clinical guidelines for $\mathrm{OAB}^{8)}$. Over recent years, the use of physiotherapy for elderly people has attracted significant attention, predominantly due to the booming healthy life style and the development of preventive medicine ${ }^{4,5)}$; however, there have been no previous reports concerning LUTS, BT, PFMT, and other types of physiotherapy with gross movement. All previous research involved local motion. Ponholzer et al. reported that LUTS scores became higher when individuals exhibited more risk factors for metabolic syndrome, such as hypertension, abnormal serum lipid levels, and high blood glucose ${ }^{9)}$. Metabolic syndrome and LUTS have many pathophysiological features in common, particularly OAB. PA in elderly people has shown beneficial changes for both weight loss and metabolic syndrome ${ }^{5)}$. These improvements of general health by PA can therefore result in beneficial changes for both LUTS and OAB. Our hypothesis was that PA for elderly people, as a preventative exercise, would improve LUTS, and the purpose of this study was therefore to examine the relationship between PA in elderly people and LUTS or OAB.

Our study identified beneficial changes in the QOL because of PA in the general elderly population. Furthermore, the outcomes of this study proved that elderly people who have LUTS and OAB could improve their symptoms because of preventative exercise. It is inferred that successive and conscious systemic physical activities, rather than "Muscle Enhancing Club" exercise program, were effective in improving blood flow in the bladder and the pelvis, which are involved in the lower urinary tract function, and in activating the nervous system. These were particularly effective for OAB, which is deemed to be involved in perception. Short duration and light intensity exercise training contributed to the prevention and treatment of 
LUTS and OAB. Even without treatment such as BT, PFMT and other types of physiotherapy, Even without treatment such as BT, PFMT and other types of physiotherapy, the conscious participation in PA programs is effective in reducing urinary symptoms. From the aspect of preventive medicine, the promotion of preventive exercise has been shown to be beneficial for LUTS. Additionally, there will be positive meaning to physical exercise classes for elderly people, which are organized by local governments. In conclusion, motivating light intensity exercise during preventive exercise classes, such as the "Muscle Enhancing Club", improve QOL, storage symptoms in LUTS, and are beneficial in the prevention and treatment of LUTS.

\section{ACKNOWLEDGEMENTS}

The authors would like to acknowledge participants of the "Muscle Enhancing Club 2010", and Ms. Hideko Ishikawa and Ms. Yukiko Akiba (Division of Welfare Health, Asahikawa City) for their contributions and for helping to collect data.

\section{REFERENCES}

1) Bø K, Talseth T: Long-term effect of pelvic floor muscle exercise 5 years after cessation of organized training. Obstet Gynecol, 1996, 87: 261-265. [Medline] [CrossRef]

2) Milne JL: Behavioral therapies for overactive bladder: making sense of the evidence. J Wound Ostomy Continence Nurs, 2008, 35: 93-101, quiz 102-103. [Medline] [CrossRef]

3) Parsons JK, Kashefi C: Physical activity, benign prostatic hyperplasia, and lower urinary tract symptoms. Eur Urol, 2008, 53: 1228-1235. [Medline] [CrossRef]

4) Fairhall N, Sherrington C, Clemson L, et al.: Do exercise interventions designed to prevent falls affect participation in life roles? A systematic review and meta-analysis. Age Ageing, 2011, 40: 666-674. [Medline] [CrossRef]

5) Stehr MD, von Lengerke T: Preventing weight gain through exercise and physical activity in the elderly: a systematic review. Maturitas, 2012 , 72 : $13-22$. [Medline] [CrossRef]

6) Cockett AT, Aso Y, Chatelain C, et al.: International consultation on benign prostatic hyperplasia, 2nd ed. Paris, 1993.

7) Homma Y, Yoshida M, Seki N, et al.: Symptom assessment tool for overactive bladder syndrome—overactive bladder symptom score. Urology, 2006, 68: 318-323. [Medline] [CrossRef]

8) Yamaguchi O, Nishizawa O, Takeda M, et al. Neurogenic Bladder Society: Clinical guidelines for overactive bladder. Int J Urol, 2009, 16: 126-142. [Medline] [CrossRef]

9) Ponholzer A, Temml C, Wehrberger C, et al.: The association between vascular risk factors and lower urinary tract symptoms in both sexes. Eur Urol, 2006, 50: 581-586. [Medline] [CrossRef] 